\title{
Lymphocyte Activation Process
}

National Cancer Institute

\section{Source}

National Cancer Institute. Lymphocyte Activation Process. NCI Thesaurus. Code C40833.

Lymphocyte Activation involves stimulation of the production and activity of

lymphocytes. Stimulation increases the immune response to invading substances. 